\title{
Epidermal growth factor receptor (EGFR) and prostaglandin-endoperoxide synthase 2 (PTGS2) are prognostic biomarkers for patients with resected colorectal cancer liver metastases
}

\begin{abstract}
J A C M Goos ${ }^{1,2}$, A C Hiemstra ${ }^{1}$, V M H Coupé ${ }^{3}$, B Diosdado ${ }^{1}$, W Kooijman ${ }^{1}$, P M Delis-Van Diemen ${ }^{1}$, C Karga $^{2}$, J A M Beliën ${ }^{1}$, C W Menke-van der Houven van Oordt ${ }^{4}$, A A Geldof ${ }^{2}$, G A Meijer ${ }^{1}$, O S Hoekstra ${ }^{2}$, R J A Fijneman ${ }^{*}, 1$ and the DeCoDe PET group ${ }^{5}$

${ }^{1}$ Department of Pathology, VU University Medical Center, CCA 1.08, De Boelelaan 1117, 1081HV Amsterdam, The Netherlands; ${ }^{2}$ Department of Radiology \& Nuclear Medicine, VU University Medical Center, CCA 1.08, De Boelelaan 1117, 1081HV Amsterdam, The Netherlands; ${ }^{3}$ Department of Epidemiology and Biostatistics, VU University Medical Center, CCA 1.08, De Boelelaan 1117, 1081 HV Amsterdam, The Netherlands and ${ }^{4}$ Department of Medical Oncology, VU University Medical Center, CCA 1.08, De Boelelaan 1117, 1081HV Amsterdam, The Netherlands
\end{abstract}

Background: Resection of colorectal cancer liver metastasis (CRCLM) with curative intent has long-term benefit in $\sim 40 \%$ of cases. Prognostic biomarkers are needed to improve clinical management and reduce futile surgeries. Expression of epidermal growth factor receptor (EGFR) and prostaglandin-endoperoxide synthase 2 (PTGS2; also known as cyclooxygenase-2) has been associated with carcinogenesis and survival. We investigated the prognostic value of EGFR and PTGS2 expression in patients with resected CRCLM.

Methods: Formalin-fixed paraffin-embedded CRCLM tissue and corresponding primary tumour specimens from a multiinstitutional cohort of patients who underwent liver resection between 1990 and 2010 were incorporated into tissue microarrays (TMAs). TMAs were stained for EGFR and PTGS2 by immunohistochemistry. The hazard rate ratio (HRR) for the association between expression in CRCLM and overall survival was calculated using a 500-fold cross-validation procedure.

Results: EGFR and PTGS2 expression could be evaluated in 323 and 351 patients, respectively. EGFR expression in CRCLM was associated with poor prognosis (HRR 1.54; $P<0.01$ ) with a cross-validated HRR of $1.47(P=0.03)$. PTGS2 expression was also associated with poor prognosis (HRR 1.60; $P<0.01$ ) with a cross-validated HRR of $1.63(P<0.01)$. Expression of EGFR and PTGS2 remained prognostic after multivariate analysis with standard clinicopathological variables (cross-validated HRR $1.51 ; P=0.02$ and crossvalidated HRR 1.59; $P=0.01$, respectively). Stratification for the commonly applied systemic therapy regimens demonstrated prognostic value for EGFR and PTGS2 only in the subgroup of patients who were not treated with systemic therapy $(\mathrm{HRR} 1.78 ; P<0.01$ and $\mathrm{HRR}$ 1.64; $P=0.04$, respectively), with worst prognosis when both EGFR and PTGS2 were highly expressed (HRR 3.08; $P<0.01)$. Expression of PTGS2 in CRCLM was correlated to expression in patient-matched primary tumours $(P=0.02,69.2 \%$ concordance).

Conclusions: EGFR and PTGS2 expressions are prognostic molecular biomarkers with added value to standard clinicopathological variables for patients with resectable CRCLM. 
Colorectal cancer (CRC) is the third most prevalent cancer type worldwide and the second leading cause of cancer mortality in developed countries, which is mainly due to hematogenous dissemination to the liver (Welch and Donaldson, 1979; Ferlay et $a l, 2010)$. Liver resection is the only intentionally curative treatment option for patients with CRC liver metastases (CRCLM). Patient eligibility relies on standard prognostic clinicopathological variables such as presence of multiple liver metastases, positive lymph nodes at the time of primary tumour resection, maximal CRCLM diameter larger than $5 \mathrm{~cm}$ and presentation of metastases within 12 months after diagnosis of the primary tumour (Nordlinger et al, 1996; Fong et al, 1999; Rees et al, 2008; Yamaguchi et al, 2008). However, 5-year survival rates hardly exceed $40 \%$, indicating the need for better prognostic biomarkers to improve clinical management of CRCLM patients (Kanas et al, 2012).

Activation of the tyrosine kinase receptor epidermal growth factor receptor (EGFR) triggers RAS/RAF/MAPK signalling and promotes proliferation, angiogenesis and metastasis, rendering EGFR as a clinical target for cancer therapy (Ciardiello and Tortora, 2008). Likewise, increased expression of prostaglandinendoperoxide synthase 2 (PTGS2) leads to increased production of $\mathrm{PGE}_{2}$, which promotes cancer cell growth through EP2 receptormediated signalling (Castellone et al, 2005). Inhibition of PTGS2, for example, by non-steroidal anti-inflammatory drugs such as aspirin, has been shown to reduce CRC incidence as well as to improve clinical outcome following CRC surgery (Tougeron et al, 2013; Zoratto et al, 2014). Importantly, there exists a complex interplay between EGFR and PTGS2 expression. EGFR activation can induce PTGS2 expression in colon cancer cells (Coffey et al, 1997). In turn, upregulation of PTGS2 leads to $\mathrm{PGE}_{2}$-mediated transactivation of EGFR (Pai et al, 2002; Buchanan et al, 2003). Both EGFR and $\mathrm{PGE}_{2}$ can activate the PI3-kinase signalling pathway. Although activating mutations in PIK3CA are potential biomarkers for resistance to treatment with anti-EGFR monoclonal antibodies in metastatic CRC, some patients with PIK3CA mutations may benefit from treatment with the PTGS2 inhibitor aspirin (Liao et al, 2012; Mao et al, 2012; Tougeron et al, 2013). These data underscore the potential biological and clinical relevance of EGFR and PTGS2 expressions in metastatic CRC. In the current retrospective study, we assessed the prognostic value of EGFR and PTGS2 expressions in CRCLM of patients who underwent liver resection with curative intent.

\section{MATERIALS AND METHODS}

Patient study population. The patient study population was selected as previously described (Goos et al, 2013). In brief, patients were identified who underwent CRCLM resection in one of the seven Dutch hospitals affiliated with the DeCoDe PET group between 1990 and 2010. Formalin-fixed paraffin-embedded (FFPE) tissue specimens were collected from one histologically confirmed CRCLM sample and adjacent control liver tissue. When available, also the corresponding primary tumour and adjacent control colon tissue were collected. Patients with multiple primary tumours were excluded. Collection, storage and use of clinicopathological data and tissue specimens were performed in compliance with the 'Code for Proper Secondary Use of Human Tissue in The Netherlands', and approved according to local and national regulations (Stichting FMWV Rotterdam, 2011).

Tissue microarrays. Tissue microarrays (TMAs) were generated as previously described (Simon et al, 2004; Goos et al, 2013). Briefly, three tissue core biopsies of $0.6 \mathrm{~mm}$ diameter were punched from morphologically representative areas of all FFPE donor blocks and transferred into TMA recipient paraffin blocks using the 3DHISTECH TMA Master (v1.14, 3DHISTECH Ltd, Budapest, Hungary).

Immunohistochemistry. TMA sections $(4 \mu \mathrm{m})$ were deparaffinised by xylene and rehydrated with decreasing alcohol series. For EGFR staining, sections were preprocessed with ER2 (Leica Biosystems, Newcastle, UK) and incubated with primary mouse monoclonal antibody directed against human EGFR $(1: 25,15 \mathrm{~min}$, Novocastra Laboratories, Newcastle, UK), followed by incubation with secondary anti-mouse antibody ( $8 \mathrm{~min}$, Novocastra Detection System, Leica Biosystems, Newcastle, UK) using BondMax Immunostainer (Menarini Diagnostics, Firenze, Italy). For PTGS2 staining, antigen retrieval was performed by microwave heating in citric acid (10 mM, pH 6.0) and endogenous peroxidase quenching in $0.3 \% \mathrm{H}_{2} \mathrm{O}_{2} /$ methanol $(25 \mathrm{~min})$. Primary rabbit polyclonal monospecific antibody directed against human PTGS2 (1:200, $1 \mathrm{~h}$, Atlas Antibodies, Stockholm, Sweden) was incubated at room temperature. Secondary anti-rabbit antibodies (Envision Plus, Dako, Heverlee, Belgium) were incubated for $30 \mathrm{~min}$. Secondary antibodies were visualised by liquid diaminobenzidine substrate chromogen system. FFPE A431 cells were stained as positive controls for EGFR expression and FFPE Caco-2 cells as positive controls for PTGS2 expression. Incubation without primary antibody served as negative control.

Evaluation of protein expression. The Mirax slide scanner system equipped with a $20 \times$ objective with a numerical aperture of 0.75 (Carl Zeiss B.V., Sliedrecht, The Netherlands) and a Sony DFWX710 Fire Wire $1 / 3^{\prime \prime}$-type progressive SCAN IT CCD (pixel size $4.65 \times 4.65 \mu \mathrm{m}$, Tokyo, Japan) was used to digitally capture the immunohistochemical stainings, as described previously (Goos et al, 2013). The actual scan resolution (effective pixel size in the sample plane) at $20 \times$ was $0.23 \mu \mathrm{m}$. Computer monitors used for image analysis were calibrated using the Spyder2PRO software (v1.0-16, Pantone Colorvision, Regensdorf, Switzerland). Frequencies of neoplastic epithelial cells expressing EGFR at the plasma membrane and PTGS2 at the nuclear membrane were scored for individual TMA core biopsies (categories $0,1-25,26-50,51-75$, 76-100\%) using dedicated TMA scoring software (v1.14.25.1, 3DHISTECH Ltd). Tissue samples were independently evaluated by a second investigator without knowledge of clinicopathological information at the time of assessment $\left(K_{\mathrm{w}, \mathrm{EGFR}}=84 \%\right.$ and $\left.K_{\mathrm{w}, \mathrm{PTGS} 2}=85 \%\right)$.

Statistical analysis. Statistical analysis was performed using IBM SPSS Statistics 20.0 software (SPSS Inc., Chicago, IL, USA) and R Statistics 3.0.1 software (RStudio Inc., Boston, MA, USA). Excluded from the analyses were patients who died within 2 months after liver resection, when no data was available on survival status or time of survival, or when tissue cores were non-evaluable due to technical reasons (Figure 1). Overall survival (OS) was defined as the time in months after surgery until death in a follow-up period of 10 years. The prognostic value of EGFR and PTGS2 expressions was assessed using a 500-fold cross-validation procedure (Goos et al, 2013). Per cross-validation cycle the study population was randomly subdivided in a training and a validation set (50\%:50\%). In each training set, the optimal cutoff for dichotomizing frequency scores into 'low' and 'high' expressions was calculated using receiver operating characteristic curve analysis for survival data with 3-year OS as the outcome of interest (Heagerty and Zheng, 2005; Zlobec et al, 2007). Frequency scores of the corresponding validation sets were dichotomised using this cutoff and a crude hazard rate ratio (HRR) was calculated in a Cox regression analysis with OS as outcome. Established clinicopathological prognostic variables were included in a multivariate Cox regression analysis (Fong et al, 1999). The average cross-validated HRR $\left(\mathrm{HRR}_{\mathrm{av}}\right)$ of the validation sets was calculated and the $P$-value of the cross-validation analysis was defined as the percentage of 
cross-validated HRRs smaller than $1\left(\mathrm{HRR}_{\mathrm{av}}<1\right)$. The relation between protein expression and OS in the total study population and a number of population subgroups was visualised by KaplanMeier curves using the most frequently selected cutoff in the crossvalidation procedure as the optimal cutoff. The potential interaction between protein expression and systemic therapy or primary tumour localisation was investigated using Cox regression. Combined prognostic values of EGFR and PTGS2 expressions were assessed by stepwise backward regression analysis with $P>0.1$ as exclusion criterion. Correlations were calculated using Pearson's correlation test. All statistical tests were two sided with $P$-values considered significant when $<0.05$. All data reported were REMARK compliant (McShane et al, 2005).

\section{RESULTS}

Demographic characteristics. Current study population consisted of 507 patients with CRCLM who were treated with liver surgery with curative intent. Cumulative 5-year OS of the study population was $41.2 \%$ (Supplementary Figure S1). Tissue specimens were available of all 507 patients, and corresponding primary tumour tissue was obtained for 234 patients. Characteristics of the patient study population are summarised in Supplementary Table S1 and described previously (Goos et al, 2013).

EGFR and PTGS2 expressions are associated with poor prognosis. Tissue specimens were immunohistochemically stained

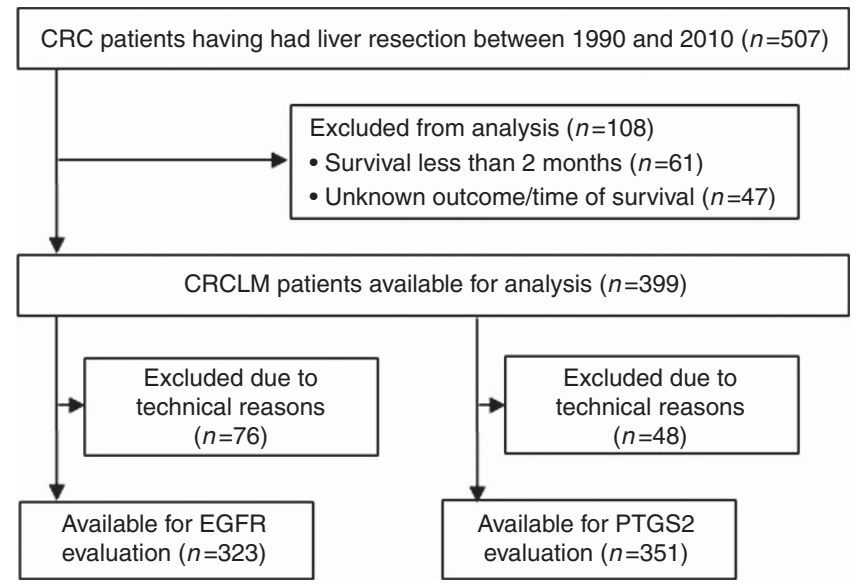

Figure 1. Schematic overview of the study cohort.
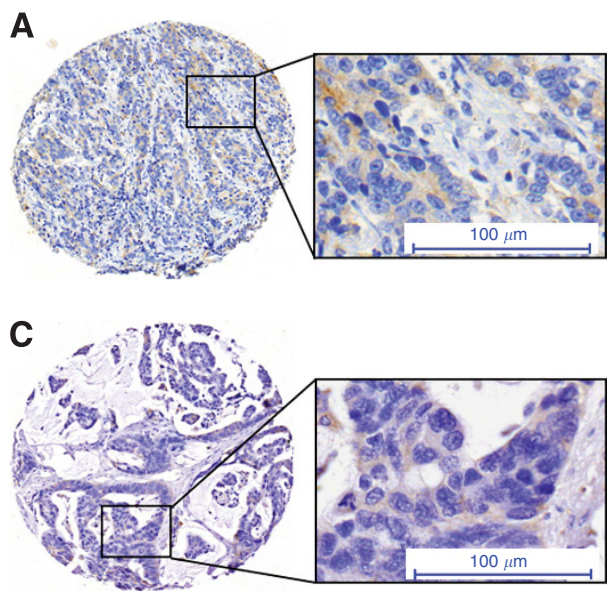

for EGFR and PTGS2 (Figure 2). EGFR was scored based on its CRCLM epithelial plasma membrane expression in 323 patients (Figure 1 and Supplementary Table S2). Patients with high EGFR expression had a significantly lower OS than patients with low EGFR expression $\left(\mathrm{HRR}_{\mathrm{av}} 1.47 ; P=0.03\right.$; Supplementary Figure S2A). The total study population was dichotomised based on EGFR expression (Supplementary Figure S3A). Median OS for patients with low EGFR expression was 58 months and for patients with high EGFR expression 40 months (HRR 1.54, 95\% CI 1.12-2.11, $P<0.01$; Figure $3 \mathrm{~A}$ ). To evaluate whether the prognostic value of EGFR expression was independent of established prognostic clinicopathological variables, a multivariate analysis was performed including EGFR protein expression, primary tumour-to-liver metastasis interval $<12$ months, number of liver metastases $>1$, maximal tumour diameter $>5.0 \mathrm{~cm}$, serum CEA level $>200 \mathrm{ng} \mathrm{ml}^{-1}$ and lymph node positivity at the time of diagnosis of the primary tumour. Also on inclusion of these variables, EGFR expression was associated with poor OS $\left(\mathrm{HRR}_{\mathrm{av}} 1.54 ; P=0.02\right.$; Supplementary Figure S2B).

PTGS2 immunohistochemical staining was scored based on its nuclear membrane expression in neoplastic CRCLM epithelium in 351 patients (Figure 1 and Supplementary Table S2). High expression of PTGS2 was associated with decreased OS $\left(\mathrm{HRR}_{\mathrm{av}}\right.$ $1.63 ; P<0.01$; Supplementary Figure S2C). On the basis of dichotomisation of PTGS2 expression (Supplementary Figure S3B), median OS was 53 months for patients with PTGS2-negative CRCLM and 32 months for patients with PTGS2-positive CRCLM (HRR 1.60, 95\% CI 1.14-2.26, $P<0.01$; Figure 3B). Multivariate analysis including clinicopathological variables showed that PTGS2 expression remained associated with poor prognosis $\left(\mathrm{HRR}_{\mathrm{av}} 1.59\right.$; $P=0.01$; Supplementary Figure S2D).

When EGFR and PTGS2 expressions were combined with standard clinicopathological variables in a multivariate analysis, both EGFR (HRR 1.91, 95\% CI 1.31-2.81, $P<0.01$ ) and PTGS2 (HRR 1.50, 95\% CI 1.00-2.26, $P=0.05$ ) were retained, indicating that they function as independent prognostic variables. Patients with high CRCLM expression of both EGFR and PTGS2 had a significantly lower OS than patients without elevated EGFR and/or PTGS2 expression (HRR 1.88, 95\% CI 1.18-2.98, $P<0.01$; Figure 3C), with a median OS difference of 43 months between patients with and without elevated levels of both EGFR and PTGS2.

Prognostic value of EGFR and PTGS2 expressions in patient subgroups. Administration of systemic therapy, pre-, peri- or postoperative to liver resection, may affect clinical outcome.
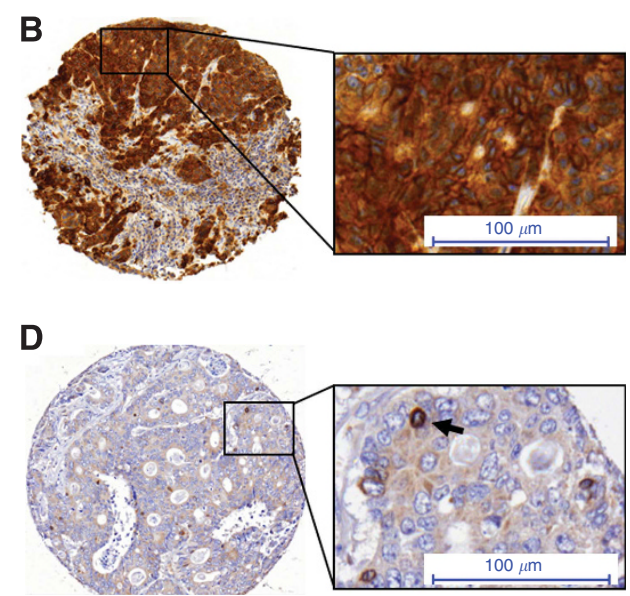

Figure 2. Staining examples of (A) low EGFR expression, (B) high EGFR expression, (C) low PTGS2 expression and (D) high PTGS2 expression (arrow) in epithelium of CRCLM. 
Therefore, the prognostic value of EGFR and PTGS2 was stratified for treatment with systemic therapy (Supplementary Table S3). High EGFR and high PTGS2 expressions were significantly associated with poor survival in patients who did not receive systemic therapy within 6 months before or following surgery (HRR 1.78, 95\% CI 1.19-2.67, $P<0.01$; Figure 4A and HRR 1.64, $95 \%$ CI $1.04-2.59, P=0.04$; Figure $4 \mathrm{~B}$, respectively). In this subgroup, patients with high CRCLM expression of both EGFR and PTGS2 had particularly poor prognosis (HRR 3.08, 95\% CI $1.66-5.73, P<0.01$; Figure 4C). However, in the subgroup of patients who did receive systemic therapy, no significant associations between OS and EGFR or PTGS2 expression were observed (HRR 1.08, 95\% CI 0.63-1.84, $P=0.78$; Figure 4D and HRR 1.27, 95\% CI 0.74-2.17, $P=0.40$; Figure 4E, respectively). Similarly, in this subgroup, combined expression of EGFR and PTGS2 lacked prognostic value (HRR 0.81, 95\% CI 0.37-1.77, $P=0.59$; Figure $4 \mathrm{~F}$ ). Notwithstanding these results in the subgroups, the interaction terms for treatment with systemic therapy and either EGFR expression $(P=0.12)$ or PTGS2 expression $(P=0.33)$ were not significant.
A

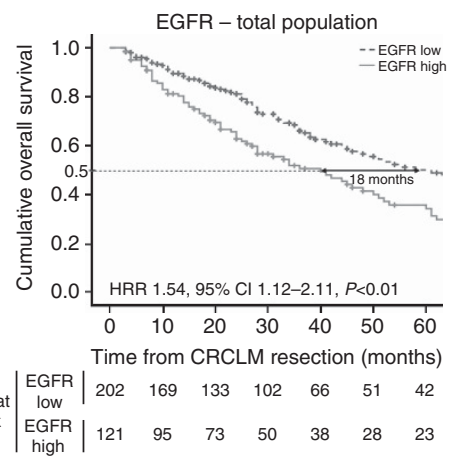

B

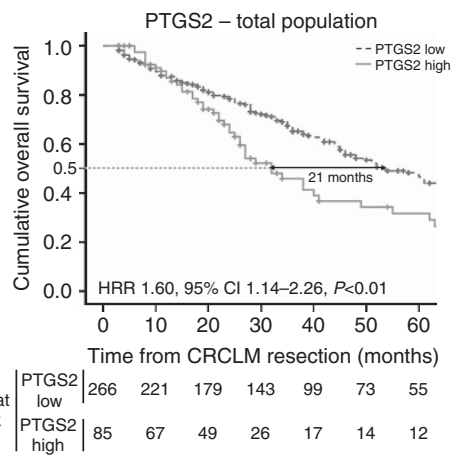

C

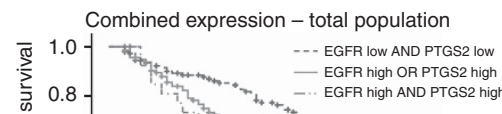
- EGFR high OR PTGS2 high

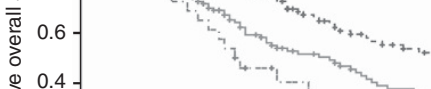

$\sum_{\substack{0 \\ 0}}^{0} 0.4$ 离 0.2

डै 0.0 - HRR $1.88,95 \%$ Cl 1.18-2.98, $P<0.01$

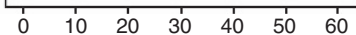
Time from CRCLM resection (months)

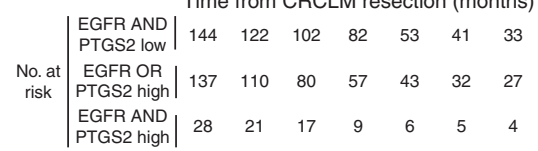

Figure 3. Kaplan-Meier graphs depicting OS in months stratified by (A) EGFR expression, (B) PTGS2 expression and (C) combined EGFR and PTGS2 expression. (A, B) HRR compares patients with high and low expression. (C) HRR compares patients in whom both EGFR and PTGS2 were highly expressed with patients in whom neither or either EGFR or PTGS2 levels were elevated.

A

EGFR - not treated with systemic therapy

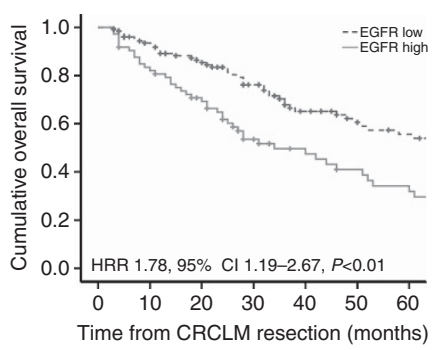

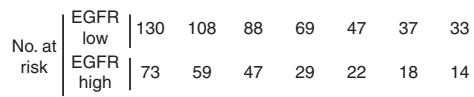

D

EGFR - treated with systemic therapy

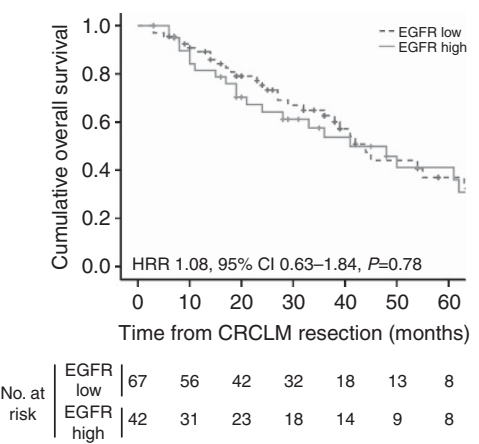

B

PTGS2 - not treated with systemic therapy

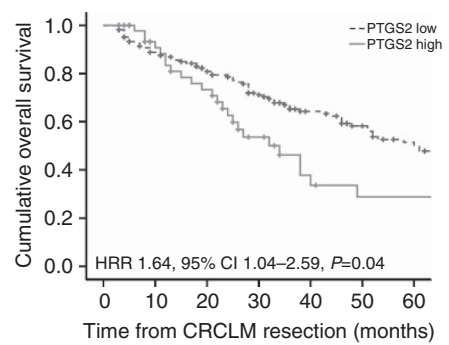

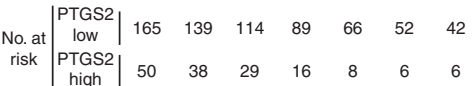

E

PTGS2 - treated with systemic therapy

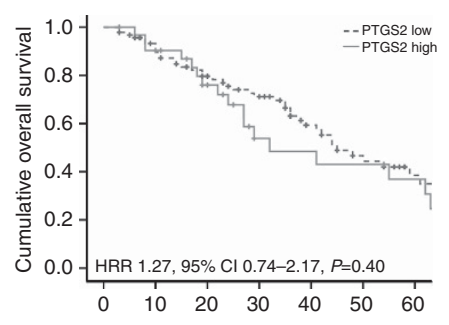

Time from CRCLM resection (months)

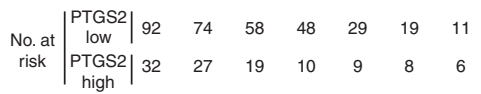

C

Combined expression not treated with systemic therapy

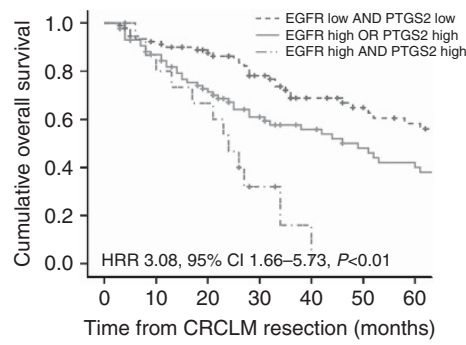

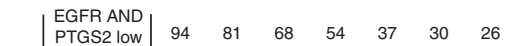

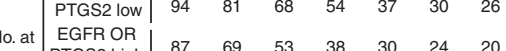
risk \begin{tabular}{l|lllllll} 
EGFR AND & 16 & 12 & 10 & 3 & 0 & 0 & 0
\end{tabular}

$\mathbf{F}$ Combined expression treated with systemic therapy

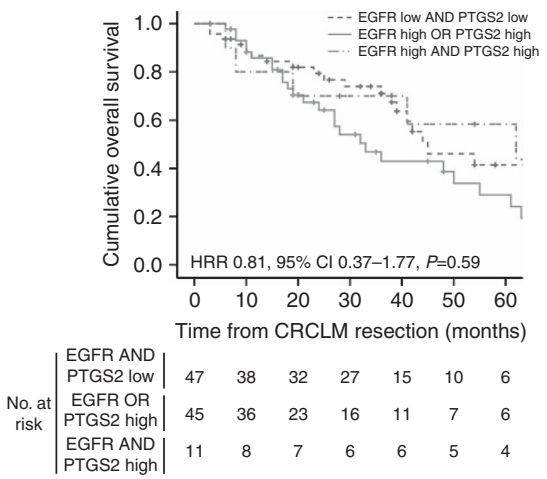

Figure 4. Kaplan-Meier graphs depicting OS in months for (A-C) patients in which CRCLM were not treated with systemic therapy and (D-F) patients in whom CRCLM were treated with systemic therapy. OS was stratified by (A, D) EGFR expression, (B, E) PTGS2 expression and (C, F) combined EGFR and PTGS2 expression. (A, B, D, E) HRR compares patients with high and low expression and (C,F) HRR compares patients in whom both EGFR and PTGS2 were highly expressed with patients in whom neither or either EGFR or PTGS2 levels were elevated. Information of systemic therapy was unavailable for $n=11$ and $n=12$ patients of which tissue samples were evaluated for EGFR and PTGS2 expression, respectively. 
The prognostic value of EGFR and PTGS2 expressions in CRCLM was also separately evaluated in colon and rectal cancer patients. High EGFR expression was associated with poor survival of colon cancer patients (HRR 1.71, 95\% CI 1.16-2.51, $P<0.01$; Supplementary Figure S4A), which was not observed for rectal cancer patients (HRR 1.19, 95\% CI 0.66-2.15, $\quad P=0.57$; Supplementary Figure S4C). PTGS2 expression was associated with decreased OS in both colon cancer patients (HRR 1.60, 95\% CI 1.06-2.42, $P=0.03$; Supplementary Figure S4B) and rectal cancer patients (HRR 1.89, 95\% CI 1.01-3.51, $P=0.05$; Supplementary Figure S4D). The interaction terms for primary tumour location and either EGFR expression $(P=0.55)$ or PTGS2 expression $(P=0.62)$ were not significant.

EGFR and PTGS2 expressions in primary CRC and corresponding CRCLM. As primary CRC tissue material is more readily available for pathological examination than CRCLM surgical specimens, we investigated whether EGFR and PTGS2 expressions in the primary tumour were correlated to their expression in patientmatched CRCLM. EGFR and PTGS2 expressions could be evaluated for 141 and 166 CRC-CRCLM pairs, respectively (Supplementary Figure S5). Expression of EGFR by primary tumours and corresponding CRCLM was inconsistent $(P=0.51)$, whereas PTGS2 expression was concordant for $69.2 \%$ of CRCCRCLM pairs $(P=0.02$; Supplementary Table S4). When patients who received systemic therapy preoperatively to primary CRC resection and/or liver resection were excluded from analysis, EGFR expression remained uncorrelated $(P=0.61)$, whereas for PTGS2 expression the proportion of concordant pairs was $70.4 \%(P<0.01$; Supplementary Table S5).

\section{DISCUSSION}

We investigated the prognostic value of EGFR and PTGS2 protein expressions in a large cohort of patients who underwent CRCLM resection with curative intent. High expression was associated with poor prognosis, with a difference in median OS between high and low expression of 18 months for EGFR and of 21 months for PTGS2. These differences are substantial, considering the median OS of the total study population of only 27 months, and correspond with the prognostic value of EGFR and PTGS2 expressions observed in earlier stages of CRC (Yamauchi et al, 2002; Soumaoro et al, 2004; Azria et al, 2005; Spano et al, 2005; Galizia et al, 2006; Ogino et al, 2008). Moreover, the difference in median OS was 43 months between patients with high expression of both EGFR and PTGS2 in CRCLM and patients with no elevated levels of EGFR and PTGS in CRCLM. Multivariate analysis demonstrated that EGFR and PTGS2 were prognostic biomarkers independent from each other and from well-known clinicopathological prognostic variables, such as positive lymph nodes at the time of primary tumour resection, presentation of metastases within 12 months after diagnosis of the primary tumour, presence of more than a single liver metastasis, high serum CEA level and maximal CRCLM diameter larger than $5.0 \mathrm{~cm}$. Microsatellite instability (MSI) is a molecular variable with prognostic value that has previously been associated with EGFR and PTGS2 expressions in primary CRC and, as such, qualifies as a potential confounding factor (Karnes et al, 1998; Ogino et al, 2006; Yuan et al, 2009; Colussi et al, 2013). The MSI status of the patients in our cohort was not known; however, as only $3 \%$ of CRCLM is estimated to be microsatellite instable (Haddad et al, 2004), the confounding effects of MSI status are presumed to have a minor role in our study population.

Approximately one-third of the patients in our study population were treated with systemic therapy during the course of their disease. Stratification for treatment revealed that the prognostic value of EGFR and PTGS2 expressions was restricted to the subgroup of patients who did not receive systemic therapy. Especially patients with CRCLM that highly expressed both EGFR and PTGS had a dismal prognosis. In contrast, EGFR and PTGS2 expressions were not associated with OS in patients who did receive systemic therapy, suggesting that EGFR- and/or PTGS2expressing tumours respond well to the commonly used 5FU-based chemotherapeutic regimens. Several inhibitors that specifically target EGFR (for example, cetuximab, panitumumab) or PTGS2 (for example, celecoxib) are being applied in clinical practice and significantly improved clinical outcome (Ciardiello and Tortora, 2008; Wang and Dubois, 2010). Dual blockade of EGFR and PTGS2 by specific inhibitors has proven effective in preclinical setting (Buchanan et al, 2007). In current study population, only a limited number of patients were treated with such inhibitors. That is, only two patients were treated with cetuximab and two patients with panitumumab. Therefore, whether these targeted drugs would be beneficial to EGFR- and/or PTGS2-expressing patients with resectable CRCLM remains to be established.

Colon and rectal cancers are frequently combined in clinical and experimental setting, as these cancer types appeared to be very similar on genomic level (Frattini et al, 2004; Cancer Genome Atlas Network, 2012). However, whether colon and rectal cancers should be considered as a single entity has often been debated. In the present study, high EGFR expression was associated with poor OS of colon cancer patients but not rectal cancer patients. This lack of prognostic value in rectal cancer patients may be explained by alteration differences in rectal cancer of genes influencing the EGFR/PI3K axis, such as PTEN, of which it has been shown that these affect survival of rectal cancer patients, but are not associated with survival in colon cancer patients (Bohn et al, 2013). PTGS2 expression was associated with poor survival in both colon and rectal cancer patients.

We also examined the correlation of protein expression between primary CRC and corresponding CRCLM, as alterations present in the primary tumour are frequently also present in the corresponding metastases (Stange et al, 2010; Knijn et al, 2011). Although PTGS2 expression in CRCLM was correlated to its expression in patient-matched primary tumours $(P=0.02)$, this was not the case for EGFR expression $(P=0.51)$. Similar differences in EGFR expression between primary CRC and CRCLM have been reported by others and could be explained by an increase of genetic alterations commonly detected during tumour progression and metastasis (Diep et al, 2006; Yarom et al, 2010). The correlation between PTGS2 expression in primary CRC and CRCLM may indicate that protein levels in CRCLM are to a certain extent predetermined by molecular alterations present in the primary CRC. Although predicting PTGS2 expression in CRCLM from its expression in the corresponding primary tumour may seem appealing, one should be cautious as still 30.8\% of PTGS2 expression scores of CRC-CRCLM pairs were discordant. The primary CRC-CRCLM correlation observed for PTGS2 expression, and the lack of it for EGFR expression, suggests that the role of PTGS2 expression in early colorectal carcinogenesis is more prominent than that of EGFR expression. This is in accordance with earlier findings that identify alterations of PTGS2 expression as early phase events and relate changes in EGFR expression to later stages of cancer development (Charalambous et al, 2003; Attolini et al, 2010).

In conclusion, EGFR and PTGS2 expressions are prognostic biomarkers for patients with resectable CRCLM, predominantly in patients not treated with systemic therapy. Further research is required to fully characterise the impact of these and other molecular alterations, such as mutation status of the predictive biomarkers KRAS, BRAF and PIK3CA (Tougeron et al, 2013), and establish optimal treatment for individual patients with CRCLM. 


\section{ACKNOWLEDGEMENTS}

Funding was supported by the Center for Translational Molecular Medicine, DeCoDe project (grant 03O-101).

\section{CONFLICT OF INTEREST}

The authors declare no conflict of interest.

\section{DECODE PET GROUP COLLABORATORS}

All collaborators of the DeCoDe PET group have contributed to data acquisition and approved the final paper after critical revision. Collaborators of the DeCoDe PET group are listed in alphabetical order per medical centre: NCT van Grieken, LR Perk, MP van den Tol, EA te Velde, AD Windhorst (VU University/VU University Medical Center - Amsterdam); J Baas, AM Rijken (Amphia Medical Center - Breda); MW van Beek, HJ Pijpers (Catharina Medical Center/PAMM Foundation - Eindhoven); H Bril, HBAC Stockmann, A Zwijnenburg (Kennemer Gasthuis/Spaarne Medical Center - Haarlem); K Bosscha, AJ van den Brule, CJ Hoekstra, JC van der Linden (Jeroen Bosch Medical Center - 's-Hertogenbosch); IH Borel Rinkes, PJ van Diest, R van Hillegersberg, O Kranenburg, MG Lam, N Snoeren (UMCU - Utrecht); IH Liem, RM Roumen, W Vening (MMC - Veldhoven).

\section{REFERENCES}

Attolini CS-O, Cheng YK, Beroukhim R, Getz G, bdel-Wahab O, Levine RL, Mellinghoff IK, Michor F (2010) A mathematical framework to determine the temporal sequence of somatic genetic events in cancer. Proc Natl Acad Sci USA 107: 17604-17609.

Azria D, Bibeau F, Barbier N, Zouhair A, Lemanski C, Rouanet P, Ychou M, Senesse P, Ozsahin M, Pelegrin A, Dubois JB, Thezenas S (2005) Prognostic impact of epidermal growth factor receptor (EGFR) expression on loco-regional recurrence after preoperative radiotherapy in rectal cancer. BMC Cancer 5: 62.

Bohn BA, Mina S, Krohn A, Simon R, Kluth M, Harasimowicz S, Quaas A, Bockhorn M, Izbicki JR, Sauter G, Marx A, Stahl PR (2013) Altered PTEN function caused by deletion or gene disruption is associated with poor prognosis in rectal but not in colon cancer. Hum Pathol 44: 1524-1533.

Buchanan FG, Holla V, Katkuri S, Matta P, Dubois RN (2007) Targeting cyclooxygenase-2 and the epidermal growth factor receptor for the prevention and treatment of intestinal cancer. Cancer Res 67: 9380-9388.

Buchanan FG, Wang D, Bargiacchi F, Dubois RN (2003) Prostaglandin E2 regulates cell migration via the intracellular activation of the epidermal growth factor receptor. J Biol Chem 278: 35451-35457.

Cancer Genome Atlas Network (2012) Comprehensive molecular characterization of human colon and rectal cancer. Nature 487: 330-337.

Castellone MD, Teramoto H, Williams BO, Druey KM, Gutkind JS (2005) Prostaglandin E2 promotes colon cancer cell growth through a Gs-axin-beta-catenin signaling axis. Science 310: 1504-1510.

Charalambous MP, Maihofner C, Bhambra U, Lightfoot T, Gooderham NJ (2003) Upregulation of cyclooxygenase-2 is accompanied by increased expression of nuclear factor-kappa B and I kappa B kinase-alpha in human colorectal cancer epithelial cells. Br J Cancer 88: 1598-1604.

Ciardiello F, Tortora G (2008) EGFR antagonists in cancer treatment. N Engl J Med 358: 1160-1174.

Coffey RJ, Hawkey CJ, Damstrup L, Graves-Deal R, Daniel VC, Dempsey PJ, Chinery R, Kirkland SC, Dubois RN, Jetton TL, Morrow JD (1997) Epidermal growth factor receptor activation induces nuclear targeting of cyclooxygenase-2, basolateral release of prostaglandins, and mitogenesis in polarizing colon cancer cells. Proc Natl Acad Sci USA 94: 657-662.

Colussi D, Brandi G, Bazzoli F, Ricciardiello L (2013) Molecular pathways involved in colorectal cancer: implications for disease behavior and prevention. Int J Mol Sci 14: 16365-16385.
Diep CB, Kleivi K, Ribeiro FR, Teixeira MR, Lindgjaerde OC, Lothe RA (2006) The order of genetic events associated with colorectal cancer progression inferred from meta-analysis of copy number changes. Genes Chromosomes Cancer 45: 31-41.

Ferlay J, Shin HR, Bray F, Forman D, Mathers C, Parkin DM (2010) Estimates of worldwide burden of cancer in 2008: GLOBOCAN 2008. Int J Cancer 127: 2893-2917.

Fong Y, Fortner J, Sun RL, Brennan MF, Blumgart LH (1999) Clinical score for predicting recurrence after hepatic resection for metastatic colorectal cancer: analysis of 1001 consecutive cases. Ann Surg 230: 309-318.

Frattini M, Balestra D, Suardi S, Oggionni M, Alberici P, Radice P, Costa A, Daidone MG, Leo E, Pilotti S, Bertario L, Pierotti MA (2004) Different genetic features associated with colon and rectal carcinogenesis. Clin Cancer Res 10: 4015-4021.

Galizia G, Lieto E, Ferraraccio F, De Vita F, Castellano P, Orditura M, Imperatore V, La Mura A, La Manna G, Pinto M, Catalano G, Pignatelli C, Ciardiello F (2006) Prognostic significance of epidermal growth factor receptor expression in colon cancer patients undergoing curative surgery. Ann Surg Oncol 13: 823-835.

Goos JA, Coupe VM, Diosdado B, Delis-van Diemen PM, Karga C, Belien JA, Carvalho B, van den Tol MP, Verheul HM, Geldof AA, Meijer GA, Hoekstra OS, Fijneman RJ. DeCoDe PET group (2013) Aurora kinase A (AURKA) expression in colorectal cancer liver metastasis is associated with poor prognosis. Br J Cancer 109: 2445-2452.

Haddad R, Ogilvie RT, Croitoru M, Muniz V, Gryfe R, Pollet A, Shanmugathasan P, Fitzgerald T, Law CHL, Hanna SS, Jothy S, Redston M, Gallinger S, Smith AJ (2004) Microsatellite instability as a prognostic factor in resected colorectal cancer liver metastases. Ann Surg Oncol 11: 977-982.

Heagerty PJ, Zheng Y (2005) Survival model predictive accuracy and ROC curves. Biometrics 61: 92-105.

Kanas GP, Taylor A, Primrose JN, Langeberg WJ, Kelsh MA, Mowat FS, Alexander DD, Choti MA, Poston G (2012) Survival after liver resection in metastatic colorectal cancer: review and meta-analysis of prognostic factors. Clin Epidemiol 4: 283-301.

Karnes WEJ, Shattuck-Brandt R, Burgart LJ, Dubois RN, Tester DJ, Cunningham JM, Kim CY, McDonnell SK, Schaid DJ, Thibodeau SN (1998) Reduced COX-2 protein in colorectal cancer with defective mismatch repair. Cancer Res 58: 5473-5477.

Knijn N, Mekenkamp LJ, Klomp M, Vink-Borger ME, Tol J, Teerenstra S, Meijer JW, Tebar M, Riemersma S, van Krieken JH, Punt CJ, Nagtegaal ID (2011) KRAS mutation analysis: a comparison between primary tumours and matched liver metastases in 305 colorectal cancer patients. Br J Cancer 104: $1020-1026$.

Liao X, Lochhead P, Nishihara R, Morikawa T, Kuchiba A, Yamauchi M, Imamura Y, Qian ZR, Baba Y, Shima K, Sun R, Nosho K, Meyerhardt JA, Giovannucci E, Fuchs CS, Chan AT, Ogino S (2012) Aspirin use, tumor PIK3CA mutation, and colorectal-cancer survival. N Engl J Med 367: $1596-1606$

Mao C, Yang ZY, Hu XF, Chen Q, Tang JL (2012) PIK3CA exon 20 mutations as a potential biomarker for resistance to anti-EGFR monoclonal antibodies in KRAS wild-type metastatic colorectal cancer: a systematic review and meta-analysis. Ann Oncol 23: 1518-1525.

McShane LM, Altman DG, Sauerbrei W, Taube SE, Gion M, Clark GM (2005) Reporting recommendations for tumor marker prognostic studies. J Clin Oncol 23: 9067-9072.

Nordlinger B, Guiguet M, Vaillant JC, Balladur P, Boudjema K, Bachellier P, Jaeck D (1996) Surgical resection of colorectal carcinoma metastases to the liver. A prognostic scoring system to improve case selection, based on 1568 patients. Association Francaise de Chirurgie. Cancer 77: 1254-1262.

Ogino S, Brahmandam M, Kawasaki T, Kirkner GJ, Loda M, Fuchs CS (2006) Combined analysis of COX-2 and p53 expressions reveals synergistic inverse correlations with microsatellite instability and $\mathrm{CpG}$ island methylator phenotype in colorectal cancer. Neoplasia 8: 458-464.

Ogino S, Kirkner GJ, Nosho K, Irahara N, Kure S, Shima K, Hazra A, Chan AT, Dehari R, Giovannucci EL, Fuchs CS (2008) Cyclooxygenase-2 expression is an independent predictor of poor prognosis in colon cancer. Clin Cancer Res 14: 8221-8227.

Pai R, Soreghan B, Szabo IL, Pavelka M, Baatar D, Tarnawski AS (2002) Prostaglandin E2 transactivates EGF receptor: a novel mechanism for 
promoting colon cancer growth and gastrointestinal hypertrophy. Nat Med 8: 289-293.

Rees M, Tekkis PP, Welsh FKS, O'Rourke T, John TG (2008) Evaluation of long-term survival after hepatic resection for metastatic colorectal cancer: a multifactorial model of 929 patients. Ann Surg 247: 125-135.

Simon R, Mirlacher M, Sauter G (2004) Tissue microarrays. Biotechniques 36: 98-105.

Soumaoro LT, Uetake H, Higuchi T, Takagi Y, Enomoto M, Sugihara K (2004) Cyclooxygenase-2 expression: a significant prognostic indicator for patients with colorectal cancer. Clin Cancer Res 10: 8465-8471.

Spano JP, Lagorce C, Atlan D, Milano G, Domont J, Benamouzig R, Attar A, Benichou J, Martin A, Morere JF, Raphael M, Penault-Llorca F, Breau JL, Fagard R, Khayat D, Wind P (2005) Impact of EGFR expression on colorectal cancer patient prognosis and survival. Ann Oncol 16: 102-108.

Stange DE, Engel F, Longerich T, Koo BK, Koch M, Delhomme N, Aigner M, Toedt G, Schirmacher P, Lichter P, Weitz J, Radlwimmer B (2010) Expression of an ASCL2 related stem cell signature and IGF2 in colorectal cancer liver metastases with 11p15.5 gain. Gut 59: 1236-1244.

Stichting FMWV Rotterdam (2011) Code Goed Gebruik van lichaamsmateriaal. www.federa.org/? $s=1 \& \mathrm{~m}=82 \& \mathrm{p}=0 \& \mathrm{v}=4 \# 827$ Stichting FMWV Rotterdam: Rotterdam, The Netherlands.

Tougeron D, Sha D, Manthravadi S, Sinicrope F (2013) Aspirin and colorectal cancer: back to the future. Clin Cancer Res 20(5): 1087-1094.

Wang D, Dubois RN (2010) The role of COX-2 in intestinal inflammation and colorectal cancer. Oncogene 29: 781-788.

Welch JP, Donaldson GA (1979) The clinical correlation of an autopsy study of recurrent colorectal cancer. Ann Surg 189: 496-502.

Yamaguchi T, Mori T, Takahashi K, Matsumoto H, Miyamoto H, Kato T (2008) A new classification system for liver metastases from colorectal cancer in Japanese multicenter analysis. Hepatogastroenterology 55: 173-178.
Yamauchi T, Watanabe M, Kubota T, Hasegawa H, Ishii Y, Endo T, Kabeshima Y, Yorozuya K, Yamamoto K, Mukai M, Kitajima M (2002) Cyclooxygenase-2 expression as a new marker for patients with colorectal cancer. Dis Colon Rectum 45: 98-103.

Yarom N, Marginean C, Moyana T, Gorn-Hondermann I, Birnboim HC, Marginean H, Auer RC, Vickers M, Asmis TR, Maroun J, Jonker D (2010) EGFR expression variance in paired colorectal cancer primary and metastatic tumors. Cancer Biol Ther 10: 416-421.

Yuan Z, Shin J, Wilson A, Goel S, Ling YH, Ahmed N, Dopeso H, Jhawer M, Nasser S, Montagna C, Fordyce K, Augenlicht LH, Aaltonen LA, Arango D, Weber TK, Mariadason JM (2009) An A13 repeat within the 3'-untranslated region of epidermal growth factor receptor (EGFR) is frequently mutated in microsatellite instability colon cancers and is associated with increased EGFR expression. Cancer Res 69: 7811-7818.

Zlobec I, Steele R, Terracciano L, Jass JR, Lugli A (2007) Selecting immunohistochemical cut-off scores for novel biomarkers of progression and survival in colorectal cancer. J Clin Pathol 60: $1112-1116$.

Zoratto F, Rossi L, Verrico M, Papa A, Basso E, Zullo A, Tomao L, Romiti A, Lo Russo G, Tomao S (2014) Focus on genetic and epigenetic events of colorectal cancer pathogenesis: implications for molecular diagnosis. Tumor Biology; doi:10.1007/s13277-014-1845-9.

This work is published under the standard license to publish agreement. After 12 months the work will become freely available and the license terms will switch to a Creative Commons AttributionNonCommercial-Share Alike 3.0 Unported License.

Supplementary Information accompanies this paper on British Journal of Cancer website (http://www.nature.com/bjc) 